\title{
Management of differentiated thyroid cancer
}

\author{
Chintamani
}

Received: 1 December 2009 / Accepted: 2 December 2009

(C) Association of Surgeons of India 2009

Excellent overall survival and lack of any prospective study makes the management of well differentiated thyroid cancers (DTC) controversial. It has therefore become necessary to tailor the management to the patient, centre and the operating surgeon. Based on various risk stratification criteria, the patients and tumours can be divided in to low- and high-risk groups and management may be standardised. Management of "high-risk patients with high-risk tumours" is relatively straightforward, i.e. total or near total thyroidectomy while low-risk patients with low-risk tumours can be managed by a relatively less aggressive approach (i.e. lobectomy or haemithyroidectomy). The controversy is about the management of intermediate groups which can not be generalised and needs to be guided by the protocols of a centre for safe optimum surgery.

The most commonly used TNM staging system has shortcomings in that it does not address the issue of histological variants such as columnar, insular and tall cell DTCs which may have worse prognosis [1-3]. It also does not consider genetic risk factors such as gender and family history. In addition, the TNM staging system does not account for molecular alterations, which at least in experimental settings have been shown to be associated with a more aggressive disease [1]. The TNM staging system only predicts cancer mortality well but not the recurrences $[1,2,4]$. For example, patients $<45$ years of age, can only be classified as stage I or II, although the survival

Chintamani

Vardhman Mahavir Medical College,

Safdarjang Hospital, New Delhi - 110 023,

India

Chintamani $(\bowtie)$

E-mail: chintamani7@rediffmail.com rates in these patients are excellent, disease recurrence is common, occurring in as many as $40 \%$ patients $<20$ years of age $[1,5]$.

The extent of surgery has not been shown to correlate well with over all survival, although there is decreased local recurrence and improved disease-free survival with total or near total thyroidectomy $[1,5]$. The article highlights the importance of ultrasound which is indeed the gold standard for evaluating thyroid nodules and nodal disease in the neck. The role of a positive fine-needle aspiration (FNA) biopsy could be significant, but the negative FNA does not rule out metastasis. It is therefore advisable to treat palpable nodes in the presence of a primary as significant.

Regarding the routine central compartment clearance, the controversy remains. In expert hands, the procedure is safe and avoids future dissection in the tracheooesophageal groove but has its morbidity (recurrent nerve and parathyroid related complications) in inexperienced hands.

There is of course no case for routine prophylactic lateral neck dissection in DTC but in the presence of clinically or radiologically apparent nodal disease, modified radical neck dissection type III is recommended. The levels I, IIB and Va are unlikely to be involved in these cancers and thus may be spared during modified neck dissections.

\section{Reference}

1. Rebecca BL (2008) Standard and emergency therapeutic approaches for thyroid malignancies. Semins Oncol 35: 298-308

2. Patel KN, Singh B (2006) Genetic considerations in thyroid cancer. Cancer Control 13:111-118

3. American Association of Clinical Endocrinocrinologists (2001) Thyroid carcinoma task force. ACE/AAES medical/ surgical guidelines for clinical practice. Management of thyroid carcinoma. Endocr Pract 7:202-220 
4. Sherman SI, Brierley JD, Sperling M, et al. (1998) Prospective multicenter study of thyroid carcinoma treatment: initial analyses of staging and outcome. National Thyroid Cancer Treatment Co-operative Study Registry Group. Cancer
83:1012-1021

5. Cooper DS, Doherty GM, Haugen BR (2006) Management guidelines for patients with thyroid nodules and differentiated thyroid cancer. Thyroid 16:109-142 